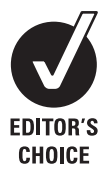

${ }^{1}$ Biomechanics Research Laboratory, School of Health Sciences, University of Wollongong, Wollongong, Australia; ${ }^{2}$ Centre for Health, Exercise and Sports Medicine, School of Physiotherapy, University of Melbourne, Melbourne, Australia

Correspondence to: Mrs Kelly-Ann Bowles, University of Melbourne CHESM/ School of Physiotherapy, Melbourne 3010, Australia; kbowles@unimelb.edu.au

Accepted 29 April 2008 Published Online First 3 June 2008

\section{What are the breast support choices of Australian women during physical activity?}

\author{
K-A Bowles, ${ }^{1,2}$ J R Steele, ${ }^{1}$ B Munro'
}

ABSTRACT

Objectives: This study aimed to quantify the breast support wearing and purchasing habits of young women and to assess factors that affect their sports bra usage during physical activity.

Design: Study results were obtained from a selfadministered mail survey, which was posted to participants after gaining their verbal consent via random telephone recruitment.

Participants: 413 women aged 20 to 35 years were recruited from New South Wales, Australia.

Results: From the returned (65\%) surveys only $41 \%$ of respondents currently wore an encapsulating sports bra during physical activity, primarily due to a lack of awareness of the importance of good breast support during physical activity. Bra size was a predictor of sports bra usage, whereby participants with large breasts were more than twice as likely to wear a sports bra as their smaller-breasted counterparts.

Conclusions: It was concluded that, although encapsulating sports bras have been shown to effectively reduce breast motion and associated exercise-induced breast discomfort, these bras were not the breast support option most commonly chosen by young women during physical activity. It is recommended that all women, irrespective of breast size, be educated on the importance of wearing a well-fitted and supportive bra during physical activity to decrease excessive strain on breast tissue structures and related breast discomfort.

With growing concerns over increasing obesity in modern society, encouraging participation in physical activity is of paramount importance. Physical activity not only lowers obesity levels through changes in metabolic rate, but also positively affects cardiovascular fitness and overall wellbeing. ${ }^{1}$ It has also been suggested that physical activity can relieve symptoms of depression and anxiety, in turn leading to a better quality of life through enhanced psychological factors such as self-concept, self-esteem and mood. ${ }^{2}$ Physical activity also assists in injury prevention, especially in women, as oestrogen and weight-bearing exercise work together to increase bone strength. ${ }^{3}$

Anecdotally, it has been suggested that some females refrain from physical activity due to the pain and/or embarrassment associated with excessive breast motion. In fact, one report found that up to $70 \%$ of female athletes complained of exercise-induced breast discomfort during exercises involving running and jumping. ${ }^{4}$ Excessive breast motion during exercise is a result of the female breast containing limited anatomical support. The skin covering breast tissue ${ }^{5}$ and the thin bands of fibrous tissue that divide the breast lobules
(Cooper's ligaments) ${ }^{6}$ provide this limited support, leaving breast tissue relatively free to move over the chest wall if unsupported, especially during movements of the torso. ${ }^{4}$ Even though the medical implications of excessive breast motion and resultant breast pain are not known, the fact that breast pain may be present during exercise suggests that some damage might be occurring to the internal breast structures. This possible breast damage, along with the negative health implications associated with a lack of physical activity due to exercise-induced breast discomfort, should cause concern to health professionals.

Due to the limited anatomical support within the female breast, external support in the form of a bra is usually recommended to reduce breast motion and associated breast discomfort Research studies have confirmed that welldesigned sports bras, such as encapsulation bras (in which each breast is supported in a separate cup; see fig 1), are more effective in limiting this motion and associated breast discomfort than standard fashion bras or crop tops, which attempt to reduce breast motion by compressing the breasts against the chest wall. ${ }^{7-11}$ Despite this research, it is not known whether women within the general community are aware of the importance of adequate breast support during exercise. In addition, no literature was found assessing sports bra usage or associated bra-related behaviours in a community-based female population. Therefore, the aim of this study was to quantify the breast support-wearing and purchasing habits of young women, focusing on breast support choices for physical activity. This included quantifying sports bra usage and other behaviours that may affect sports bra purchasing, such as age, bra size, participation in physical activity and breast pain occurrence.

\section{METHODS}

\section{Survey development}

Due to the sensitive nature of assessing underwear preferences, and with the aim of gaining information from a broad geographic area, a self-administered mail survey was used to gather data. ${ }^{12-14}$ The survey was developed with assistance from an epidemiologist and bra industry representatives, and content validity was established through focus groups composed of women in the target population, described below. The 31-question survey, including both closed and open-ended items, sought information on each respondent's demographics, bra purchasing habits and fitting history, occurrence of breast pain, physical activity participation, breast support worn during physical activity, sports bra use 


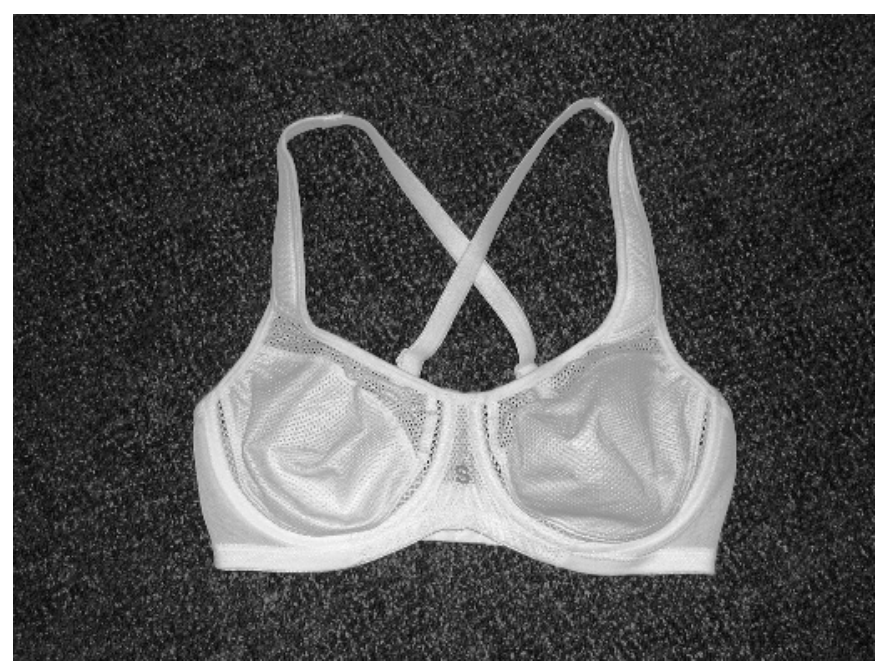

Figure 1 An encapsulating sports bra in which each breast is supported by a separate cup.

and factors affecting why the respondents did or did not wear a sports bra.

Test-retest analysis, completed on all non-time related questions, deemed the survey was reliable $(r=0.72, p<0.05) .{ }^{12}$ Full approval of the content and conduct of the survey was received from the University of Wollongong Human Research Ethics Committee prior to implementation of the survey.

\section{Survey implementation}

Women aged 20 to 35 years who resided in New South Wales (NSW), Australia, were targeted as potential study respondents as they were deemed a good representative sample of the Australian female population due to the diverse socioeconomic, cultural and geographic regions throughout NSW.

In order to gain weighted information across the broad geographical area of NSW, a stratified sampling technique was performed, with subgroups formed on the basis of Telstra White Pages telephone directory zones (Sensis, Australia) and regions of the Australian Bureau of Statistics' estimated resident population census. ${ }^{12-15}$ Using Cochran's Formula for Sampling Proportions ${ }^{16}$ it was determined that 100 respondents were required to gain an adequate sample size with a standard error of 0.05 . However, this number was increased (see Results section) to ensure sufficient subjects in each subgroup were sampled, within the time and financial constraints of the study.

To identify potential respondents who fulfilled the subject selection criteria, to obtain verbal consent to send a survey package, and to promote a higher survey return rate, contact was made via telephone, using a set script to ensure uniformity in response to each potential subject, before distributing the surveys. ${ }^{1417} 18$ Prospective telephone numbers were generated using a random numbers generation with a uniform distribution (Microsoft Excel, Microsoft Corporation, USA). Data were generated for page number, row number and column number for each White Pages telephone directory.

If a prospective respondent provided verbal consent to participate in the survey, their postal address details were confirmed and a questionnaire, written consent form, letter of explanation and stamped self-addressed envelope for survey return were mailed to the address. A non-monetary incentive of entry into a random draw to win $\$ 200$ worth of lingerie was offered to the respondents when the survey was returned to the researchers to increase response rate. ${ }^{13} 1417-19$ The brand of lingerie was not specified in order to reduce any brand bias.

A total of 4413 telephone calls were made over 12 months by a team of seven trained research assistants. From these calls, 480 potential respondents were identified and 413 of these respondents volunteered to participate in the study and were mailed a survey package. A follow-up reminder letter and complete survey package were mailed to each respondent who had not returned the survey after 4 weeks. No further follow-up was completed due to restrictions imposed by the Human Research Ethics Committee.

Of the 413 survey packages mailed to consenting respondents, 267 questionnaires were completed and returned by respondents who fulfilled the subject selection criteria $(65 \%$ response rate). This response rate was considered high for a mail survey as traditional mail surveys typically have an average response rate of below 20\%. ${ }^{19}$ A further seven questionnaires returned for analysis were completed by respondents outside the set age range and were therefore excluded from further analysis. The average age of survey respondents was 27.2 (SD 4.9) years with 101 (38\%) respondents aged 20-24 years, 67 (25\%) respondents aged 2529 years and 99 (37\%) respondents aged 30 plus years. Age groups were set in accordance with Australian Bureau of Statistics age groups for explaining population demographics. ${ }^{15}$

\section{Survey analysis}

Bivariate analysis was completed on each survey component, investigating relationships between bra size and age using cross tabulations and $\chi^{2}$ analysis (SPSS 12.0.1, SPSS Inc., USA). All data were grouped into categories to ensure assumptions of the $\chi^{2}$ analysis (including a minimum expected frequency above five) were met. ${ }^{20}$ Physical activities were coded according to the amount of vertical breast motion that might occur during that activity using the Berlei grading system. ${ }^{21}$ Activities that typically result in the least to greatest amount of vertical breast motion were coded as Factor 1 to Factor 3 activities, respectively.

To determine which variables might significantly predict sports bra usage, multivariate analysis via a logistic regression was performed. A forced-entry model for demographic predictors of sports bra usage was developed reporting odds ratios and $95 \%$ confidence intervals. In all analyses, statistical outcomes were deemed significant at an alpha level of $p \leqslant 0.05$

\section{RESULTS}

\section{Bra size}

Respondents reported 26 bra sizes, ranging from metric size $10 \mathrm{~A}$ to $18 \mathrm{H} / 20 \mathrm{DD}$ (imperial size $\sim 32 \mathrm{~A}$ to $40 \mathrm{H} / 42 \mathrm{DD}$ ). The most common size reported by respondents was $12 \mathrm{~B}$ (imperial size $\sim 34 \mathrm{~B} ; 23 \%$ ). When the bra sizes were grouped into small (A and $\mathrm{B}$ cup) and large ( $\geqslant \mathrm{C}$ cup)-breasted categories, ${ }^{7}$ $^{22} 114(43 \%)$ respondents were classified as small-breasted and $150(56 \%)$ respondents were classified as large-breasted, with three respondents not reporting a bra size (see table 1). Age and bra size were significantly related, with respondents aged 25-29 years less likely to be small-breasted than would be expected $\left(\chi^{2}=6.55 ; p=0.04\right.$; see table 1$)$. However, it should be noted that bra sizes were self-reported and, as $70-100 \%$ of females reportedly wear the incorrect size bra, ${ }^{23}$ the bra size data should be interpreted with caution. 
Table 1 Number of respondents according to age group and bra size categories $(n=267)$

\begin{tabular}{lccll}
\hline Age group & $\begin{array}{l}\text { Small- } \\
\text { breasted (\%) }\end{array}$ & $\begin{array}{l}\text { Large- } \\
\text { breasted (\%) }\end{array}$ & $\begin{array}{l}\text { Size not } \\
\text { provided (\%) }\end{array}$ & Total \\
\hline $20-24 \mathrm{yr} \mathrm{( \% )}$ & $47(18)$ & $53(20)$ & $1(0)$ & $\mathbf{1 0 1 ( 3 8 )}$ \\
$25-29 \mathrm{yr}(\%)$ & $20(7)$ & $47(18)$ & $0(0)$ & $\mathbf{6 7}(\mathbf{2 5 )}$ \\
$30-35 \mathrm{yr} \mathrm{( \% )}$ & $47(18)$ & $50(19)$ & $2(1)$ & $\mathbf{9 9}(\mathbf{3 7 )}$ \\
Total & $\mathbf{1 1 4 ( 4 3 )}$ & $\mathbf{1 5 0 ( 5 6 )}$ & $\mathbf{3 ( 1 \% )}$ & $\mathbf{2 6 7}(\mathbf{1 0 0})$ \\
\hline
\end{tabular}

\section{Sports bra usage}

Although $71 \%$ of respondents indicated that they had worn an encapsulating sports bra at some time, only $41 \%$ of respondents indicated that they currently wore an encapsulating sports bra during physical activity. Age group $\left(\chi^{2}=10.58\right.$; $\left.p<0.01\right)$ and bra size $\left(\chi^{2}=13.38 ; p<0.01\right)$ were both significantly related to respondents ever wearing a sports bra during physical activity, with only bra size significantly related to current sports bra use $\left(\chi^{2}=11.49 ; \mathrm{p}<0.01\right)$. That is, respondents aged $25-29$ years had worn a sports bra at some time during physical activity more frequently than would be expected, as did the respondents with large breasts, and a greater number of respondents with large breasts currently wore a sports bra than expected. It was interesting to note that bra size was significantly related to the correct identification of a sports bra $\left(\chi^{2}=13.33\right.$; $\left.p<0.01\right)$, as women with large breasts more readily correctly identified the encapsulating sports bra as a sports bra (see fig 1) than their small-breasted counterparts.

Perhaps the most pertinent question in the survey asked why respondents did not currently wear an encapsulating sports bra during physical activity. The most common responses to this question included "I do not feel I need to" (27\%) and "I have not considered wearing one" (15\%).

\section{Breast pain}

One hundred and seventy-one (64\%) respondents indicated that they suffered from breast pain at some time, with $45 \%$ of these respondents indicating that they believed this pain was due to menstruation. Bra size and age were not significantly related to breast pain (bra size: $\chi^{2}=1.11 ; p=0.29$, age: $\chi^{2}=0.28$; $p=0.87$ ) or the believed cause of the breast pain (bra size: $\chi^{2}$ $=1.67 ; \mathrm{p}=0.64$, age: $\left.\chi^{2}=12.02 ; \mathrm{p}=0.06\right)$.

\section{Physical activity}

Most respondents (41\%) reported their activity level as "moderately active". However, there were no significant relationships between breast size $\left(\chi^{2}=1.59 ; p=0.45\right)$ or age $\left(\chi^{2}=3.86 ; p=0.43\right)$ and the reported level of physical activity. In addition, despite $60 \%$ of respondents indicating they participated in activities typically associated with high levels of vertical breast motion (Factor 3 activities), bra size $\left(\chi^{2}\right.$ $=0.13 ; p=0.72$ ) was not significantly related to involvement in Factor 3 activities. However, a significant relationship was found between age group and involvement in Factor 3 activities $\left(\chi^{2}=21.44 ; p<0.01\right)$, whereby the older women (30-35 years) did not report participating in activities typically associated with large amounts of vertical breast displacement (43\%) as much as their younger counterparts (20-24 years; 76\%). When asked what they mostly used for breast support during the reported physical activities, 88 respondents (34\%) indicated that they wore a fashion bra, whereas 84 respondents (32\%) reported wearing a sports bra. Bra size and age were not significantly related to breast support choices during the physical activities $\left(\chi^{2}=6.43 ; p=0.09: \chi^{2}=7.32 ; p=0.29\right.$, respectively $)$, suggesting that, overall, women were slightly more likely to wear a fashion bra during physical activity than the more researchrecommended sports bra, regardless of their age or bra size.

\section{Sports bra features}

Although fit (93\%), stopping breast motion (71\%) and the presence of underwire $(45 \%)$ were current sports bra features classified by the respondents most frequently as "very important", straps slipping off the shoulder (34\%) and straps cutting into the shoulder (34\%) were the main sports bra features respondents "extremely disliked". Age was not significantly related to the importance or disliking of any sports bra feature. However, there was a significant relationship between bra size and several sports bra features including fit $\left(\chi^{2}=5.05 ; p=0.03\right)$, presence of underwire $\left(\chi^{2}\right.$ $=10.52 ; \mathrm{p}<0.01)$ and stopping breast motion $\left(\chi^{2}=10.92\right.$; $\mathrm{p}<0.01)$. That is, women with large breasts reported these three features as very important more readily than their small-breasted counterparts. With regard to disliked features, bra size was significantly related to the sports bra "creeping up" $\left(\chi^{2}=6.70\right.$; $\mathrm{p}=0.04$ ), with small-breasted subjects reporting this as a disliked feature more frequently than their large-breasted counterparts.

\section{DISCUSSION}

Previous research has found encapsulating sports bras to be more effective in limiting breast motion and associated breast pain than fashion bras or crop tops. ${ }^{8}$ However, fewer than half of the present respondents indicated they currently wore an encapsulating sports bra during physical activity, despite the fact that more than half of the respondents were classified as large-breasted (see table 1). Age group and bra size were both significantly related to respondents ever wearing a sports bra during physical activity, whereby respondents aged 25-29 years had worn a sports bra at some time during physical activity more frequently than would be expected, as had respondents with large breasts. These results are consistent with the findings that respondents aged 25-29 years were classified as large-breasted more frequently than would be expected. Interestingly, only bra size was significantly related to current sports bra use. Although it is possible that respondents with large breasts are seeking good breast support during physical activity, women with small breasts have been shown to experience $8 \mathrm{~cm}$ of vertical nipple displacement during running when unsupported. Furthermore, the finding that $35 \%$ of the respondents with large breasts who did not wear a sports bra during physical activity indicated that they "had not considered wearing one" suggests that not all young women are aware of the importance of adequate external breast support during physical activity. This result reinforces the need to educate all women on the importance of good breast support during physical activity. In designing and implementing these education programmes, factors such as the age group and bra size of the target audience should be considered.

Apart from educating women on the benefits of wearing good breast support during physical activity, education should also focus on features of a good supportive sports bra. There are currently no guidelines or standards that a bra must meet to be classified as a sports bra. In fact, less supportive crop tops ${ }^{7}$ are frequently marketed as sports bras, with $20 \%$ of respondents in the present study reporting that they believed a crop top was a sports bra. It is therefore recommended that international standards be developed so that women can more easily identify the bras that will provide adequate support during physical activity.

Although the present results suggest that breast pain is experienced by women regardless of bra size or age, despite $60 \%$ 


\section{What is already known on this topic}

As the female breast contains no supportive muscle or bone breast tissue is relatively free to move over the chest wall, especially during motion of the torso. Previous research suggests that encapsulating sports bras can decrease excessive breast motion and related breast pain, especially during physical activity.

\section{What this study adds}

This study quantifies sports bra usage in the general female population and provides insight into the reasoning behind a woman's decision whether or not to wear a sports bra during physical activity. This information is useful to all sports medicine/ sport science professionals in planning future breast health education programmes.

of respondents indicating they participated in Factor 3 activities, only nine respondents ( $5 \%$ of those who did suffer from breast pain) indicated that their perceived breast pain was due to excessive breast motion. This finding is contradictory to previous research where up to $70 \%$ of women who participated in running and jumping activities reported exercise-induced breast pain. ${ }^{4}$ The fact that most respondents in the present study reported being involved only in moderate-level activity, with older women (30-35-years) participating less in activities typically associated with large amounts of vertical breast displacement (Factor 3 activities) than their younger counterparts, may be a possible explanation for the discrepancy between the two studies. Interestingly, the fact that bra size was not significantly related to either the types of activities performed or the level of physical activity was a positive finding, as these results negate the anecdotal suggestion that breast size can impede participation in physical activity. However, the finding that $60 \%$ of respondents were participating in Factor 3 activities although not wearing a sports bra is alarming considering the negative consequences potentially associated with poor breast support during physical activity.

This study also highlighted the different requirements women have from a sports bra depending on their breast size. That is, women with large breasts had a preference for underwire in their sports bra, and more readily recognised the importance of the bra's ability to reduce breast motion.

\section{CONCLUSION}

The results of this study suggest that encapsulating sports bras are not the most common choice of breast support during physical activity for young women. Although respondents with large breasts were more likely to wear a sports bra during physical activity than their small-breasted counterparts, it appears that scientific results highlighting the greater ability of encapsulating sports bras to reduce excessive breast motion during physical activity have not reached this population. Interestingly, the reasons respondents did not wear a sports bra were not size-specific; neither was the occurrence of breast pain nor the types of physical activities women were involved in.
However, as the medical implications of poor breast support during physical activity are not well-documented, and as large amounts of breast motion can occur in women both with large and with small breasts, these findings reinforce the need to educate all women, irrespective of breast size, on the importance of wearing a well-fitted and supportive bra during physical activity. In designing and implementing these education programmes, factors such as the age and bra size must be taken into consideration, as these factors affect the bra usage and purchasing habits of young women.

Acknowledgements: The researchers would like to acknowledge the support of the Australian Research Council for their financial support of this project as part of a Strategic Partnership and Industry Training grant. The researchers would also like to acknowledge the support of Berlei, the Industry Partner for this project.

Competing interests: None declared.

Ethics approval: This work was approved by the Human Research Ethics Committee at the University of Wollongong.

Patient consent: Obtained.

\section{REFERENCES}

1. Powers SC, Howley ET. Exercise Physiology: Theory and Applications to Fitness and Performance. 2nd edn. Dubuque: Wm. C. Brown Communication Inc.,1994.

2. National Center for Chronic Disease Prevention and Health Promotion Physical Activity and Health: Surgeon General's Report on Physical Activity and Health (No. 7864). Atlanta: 1999.

3. Heaney RP, Recher RR, Saville PD. Menopausal changes in calcium balance performance. In: Bar-Or 0, Lamb D, Clarkson P, eds. Exercise and the Female - A Life span Approach. Carmel: Cooper Publishing Company,1978.

4. Gillette J. When and where women are injured in sports. Phys Sports Med 1975;3:61-70.

5. Hindle WH. The breast and exercise. In: Hale RW, ed. Caring for the Exercising Woman. New York: Elsevier Science Publishing, 1991.

6. Gehlsen G, Stoner LJ. The female breast in sport and exercise. In: Adrian MJ, ed. Sports Woman. Basel: Karger, 1987.

7. Mason BR, Page K-A, Fallon K. An analysis of movement and discomfort of the female breast during exercise and the effects of breast support in three case studies. J Sci Med Sport 1999;2:134-44.

8. Verscheure SK. How effective are sports bras designs in attenuating forces during jumping [unpublished MSc thesis]. University of Oregon, Eugene, 1999.

9. Shivitz NL. Adaptation of vertical ground reaction force due to changes in breast support in running [unpublished MSc thesis]. Oregon State University, 2001.

10. Boschma ALC. Breast support for the active woman: Relationship to 3D kinematics of running [unpublished MSc thesis]. Oregon State University, 1994.

11. Page KA, Steele JR. Breast motion and sports bra design. Implications for future research. Sports Med 1999;27:205-11.

12. Burns RB. Introduction to research methods. 2nd edn. Melbourne, Australia: Longman Cheshire, Pty. Ltd, 1994.

13. Van Kenhove P, Wijnen K, De Wulf K. The influence of topic involvement on mailsurvey response behaviour. Psychology and Marketing 2002;19:293-301.

14. National Computer Systems. Research Notes. Available at: http://www.ncs.com/ ncscorp/research (accessed 4 August 1997).

15. Australian Bureau of Statistics. 1996 Census of Population and Housing. Basic Community Profile. ABS Catalogue No. 2901.0. Australian Bureau of Statistics, 2000.

16. Cochran WG. Sampling Techniques. 2nd edn. New York: John Wiley and Sons, 1953.

17. Erwin WJ, Wheelright LA. Improving mail survey response rates through the use of monetary incentive. Journal of Mental Health Counselling 2002;24:247-55.

18. Bright KD, Smith PM. The use of incentives to affect response rates for a mail survey of U.S. marina decision makers. Forest Products Journal 2002;52:26-9.

19. Harbaugh R. Proven lessons for generating good mail survey response rates. Med Mark Media 2002;37:70-6.

20. Jaccard J, Becker MA. Statistics for the behavioural sciences. 2nd edn. Belmont, California: Wadsworth Publishing Company, 1990.

21. Berlei Grading system. Available at: http://www.berlei.com.au/About-Berlei/Berleiand-the-AIS (accessed 18 September 2007).

22. Lorenzten D, Lawson L. Selected sports bras: A biomechanical analysis of breast motion while jogging. Phys Sports Med 1987;15:128-39.

23. McGhee DE, Steele JR. How do respiratory state and measurement method affect bra size calculations? Br J Sports Med 2006;40:970-4. 